\title{
Performance of Power Systems under Sustained Random Perturbations
}

\author{
Humberto Verdejo, ${ }^{1}$ Wolfgang Kliemann, ${ }^{2}$ and Luis Vargas ${ }^{3}$ \\ ${ }^{1}$ Departamento de Ingeniería Eléctrica, Universidad de Santiago de Chile, 9170124 Santiago, Chile \\ ${ }^{2}$ Department of Mathematics, Iowa State University, Ames, IA 50011, USA \\ ${ }^{3}$ Departamento de Ingeniería Eléctrica, Universidad de Chile, 8370451 Santiago, Chile
}

Correspondence should be addressed to Humberto Verdejo; humberto.verdejo@usach.cl

Received 13 June 2014; Revised 6 October 2014; Accepted 8 October 2014; Published 5 November 2014

Academic Editor: Weihai Zhang

Copyright (C) 2014 Humberto Verdejo et al. This is an open access article distributed under the Creative Commons Attribution License, which permits unrestricted use, distribution, and reproduction in any medium, provided the original work is properly cited.

\begin{abstract}
This paper studies linear systems under sustained additive random perturbations. The stable operating point of an electric power system is replaced by an attracting stationary solution if the system is subjected to (small) random additive perturbations. The invariant distribution of this stationary solution gives rise to several performance indices that measure how well the system copes with the randomness. These indices are introduced, showing how they can be used for the optimal tuning of system parameters in the presence of noise. Results on a four-generator two-area system are presented and discussed.
\end{abstract}

\section{Introduction}

During operation, power systems encounter a variety of perturbations, most of which are properly modeled as random. This includes randomness due to consumer behavior as well as faults that produce generator outages, changes in the topology of networks, and equipment behavior. Today basically all probabilistic analyses of power system behavior address contingency and security/reliability analysis. A few papers deal with small signal analysis under random perturbations, studying stability criteria or noise-induced chaos [1].

Random perturbations act on power systems in different ways (see $[2,3]$ ), depending on whether they occur in generation, transmission, or load components. Mathematically, this means that a random process may act additively or multiplicatively on the system. For example, when loads vary in a random fashion, the system may be modelled with an additive perturbation. On the other hand, perturbations at generators lead to random factors, that is, to multiplicative noises which can be analyzed via small stability studies [4]. In this paper we show that additive noise destroys the stable operating point, replacing it with an attracting stationary and ergodic solution. The invariant distribution of this "stationary state" gives rise to performance assessments that indicate how well the system copes with the randomness. These indices include the diameter of the "loss range" (the set over which the system response varies), the total deviation of the rotor angles due to randomness, and the loss of power generation.

The goal of this assessment is to optimally tune the system parameters (such as gains and time constants of exciters or power system stabilizers) so that the loss due to random perturbations is minimized. Although the proposed technique is described in general terms, this paper concentrates on power system stabilizer (PSS) gain parameters. We base our assessment on the system linearized about a (stable) operating point, which is a good approximation to the full nonlinear model for small perturbations. For a realistic and flexible noise model we consider bounded, stationary functions of a Markov diffusion process and analyze the system response as the statistics and size of the noise vary. The applications studied here involve a four-generator two-area test system [5]. Of course, in applications to actual systems, the statistics of the noise process have to be estimated from actual system data, such as flow at load buses. 


\section{Indicators of System Performance}

2.1. Mathematical Background. In this section we describe a general methodology to assess the influence of (additive) random perturbations on the performance of a (linearized) power system. We start from the nonlinear system equations

$$
\dot{y}=f(y, p)
$$

in the state space $\mathbb{R}^{N}$, where $N_{1}$ states describe the (relative) rotor angles $\left(\delta_{1}, \ldots, \delta_{n}\right)$ and velocities $\left(\omega_{1}, \ldots, \omega_{n}\right)$, and $N_{2}$ states describe the controls $\left(N_{1}+N_{2}=N\right)$. The vector $p \in$ $\mathbb{R}^{r}$ is the vector of control parameters that can be tuned to provide optimal system performance.

Let $y^{*} \in \mathbb{R}^{N}$ be a fixed point (operating point) of system (1), and denote the linearized system at $y^{*}$ by

$$
\dot{x}=A(p) x .
$$

Throughout this section we assume that the system parameters are set such that the origin is an (exponentially) stable point of the linear system equation (2).

Persistent stochastic disturbances that lead to an additive perturbation result in the system model:

$$
\dot{x}=A(p) x+B \xi_{t},
$$

where $\xi_{t}$ is a random process with values in some set $U \subset \mathbb{R}^{m}$, and $B$ is an $N \times m$ matrix describing how the noise affects the system states. For a realistic and flexible noise model we consider the following.

(A) The background noise $\eta_{t}$ is a given stationary, ergodic Markov diffusion process defined on a suitable probability space $(\Omega, F, \mathbb{P})$ with values in a smooth manifold $M$ of finite dimension. The parameters of $\eta_{t}$ must be estimated from actual noise data, for example, via its stationary distribution, power spectrum, or autocorrelation function. A typical example of a background noise is, for example, a multidimensional Ornstein-Uhlenbeck process as follows:

$$
d \eta_{t}=C \eta_{t} d t+D d W_{t} \text { in } \mathbb{R}^{k}
$$

where $C$ is a stable $k \times k$ matrix, $W_{t}$ is a standard Wiener process, and $D$ is a matrix of suitable dimension.

(B) The system perturbation $\xi_{t}$ is a smooth, bounded function $f\left(\eta_{t}\right)$ of the background noise, and $U$ is a compact, convex neighborhood of the origin $0 \in \mathbb{R}^{m}$. The function $f: M \rightarrow U \subset \mathbb{R}^{m}$ allows us to adjust the statistics and range of the background noise $\eta_{t}$ so that one can test the system (3) with various different random perturbations. A typical example of the function $f$ is, for example, $f\left(\eta_{t}\right)=\sin \left(\eta_{t}\right)$, where $\eta_{t}$ is a one-dimensional Ornstein Uhlenbeck process, and $\sin \left(\eta_{t}\right)$ is its projection onto $[-1,1]$.

(C) To measure the influence of the size of the noise range on system performance, we introduce a parameter $\rho \geq 0$ and consider the family of functions $f^{\rho}: M \rightarrow$ $U^{\rho} \subset \mathbb{R}^{m}$ defined by $f^{\rho}(\eta)=\rho \cdot f(\eta)$. For $\rho=0$ this corresponds to the unperturbed system (2).
For the following analysis we assume the following.

(1) Matrix $A(p)$ is stable for all relevant values of the tuning parameter $p$; that is, all eigenvalues of $A(p)$ have negative real parts

(2) Matrix $\left(B, A B, A^{2} B, \ldots, A^{N-1} B\right)$ has full rank $N$.

(3) The background noise $\eta_{t}$ is the stationary solution of a stochastic differential equation on the $C^{\infty}$-manifold $M$ given by

$$
d \eta_{t}=Y_{0}\left(\eta_{t}\right) d t+\sum_{i=1}^{l} Y_{i}\left(\eta_{t}\right) \circ d W_{t}
$$

with $C^{\infty}$-vector fields $Y_{0}, \ldots, Y_{l}$. Here “o" denotes the Stratonovic stochastic differential. We assume that (5) has a unique stationary and ergodic solution with invariant distribution $\nu$ on $M$, which is guaranteed, for example, by the weak nondegeneracy condition

$$
\operatorname{dim} \mathscr{L} \mathscr{A}\left\{Y_{i}, i=1, \ldots, l\right\}(y)=\operatorname{dim} M,
$$

for all $y \in M$, where $\mathscr{L} \mathscr{A}$ denotes the Lie algebra generated by the $Y_{i}$ vectors fields.

(4) The function $f: M \rightarrow U$ is a continuous map onto the set $U \subset \mathbb{R}^{m}$ such that there exists a closed, connected subset $L \subset M$ on which the restriction $\left.f\right|_{L}$ is $C^{1}$ and the Jacobian $D f(\eta)$ has full rank for all $\eta \in L$ with $f(\eta) \in \operatorname{int} U$, the interior of $U$. As before, we assume that $U$ is a convex, compact neighborhood of the origin $0 \in \mathbb{R}^{m}$.

Under these conditions one obtains the following result.

Theorem 1. Consider the linear stochastic system (3) under assumptions (1)-(4).

(1) The system has a unique stationary and ergodic solution $x^{*}(t, p, \omega)$ for all $t \geq 0$. Its invariant distribution $\mu_{p}$ has support in a compact set $C_{p} \subset \mathbb{R}^{N}$.

(2) The stationary distribution is globally attracting; that is, let $x^{0} \in \mathbb{R}^{N}$ be an initial value for (3); then the corresponding solution $x^{*}\left(t, p, \omega, x^{0}\right)$ will enter into set $C_{p}$ in finite time and then converge in distribution to the invariant distribution $\mu_{p}$.

The proof of this Theorem is shown in the appendix.

The stationary distribution of the linearized system (3) as described in Theorem 1 is, together with the ergodic theorem, the basis for the performance indicators developed in the next section. This distribution $\mu_{p}$ and its support $C_{p}$ are related to the dynamics of the nonlinear system $\dot{y}=f(y, p)+$ $B \xi_{t}$ around the operating point $y^{*} \in \mathbb{R}^{N}$ in the following way: since the fixed point $y^{*}$ is assumed to be exponentially stable, a small additive perturbation will result (under mild conditions) in a unique stationary and ergodic solution of the nonlinear stochastic system around $y^{*}$, compared with Theorem 4.7.11 in [6]. This means that global bifurcations of 


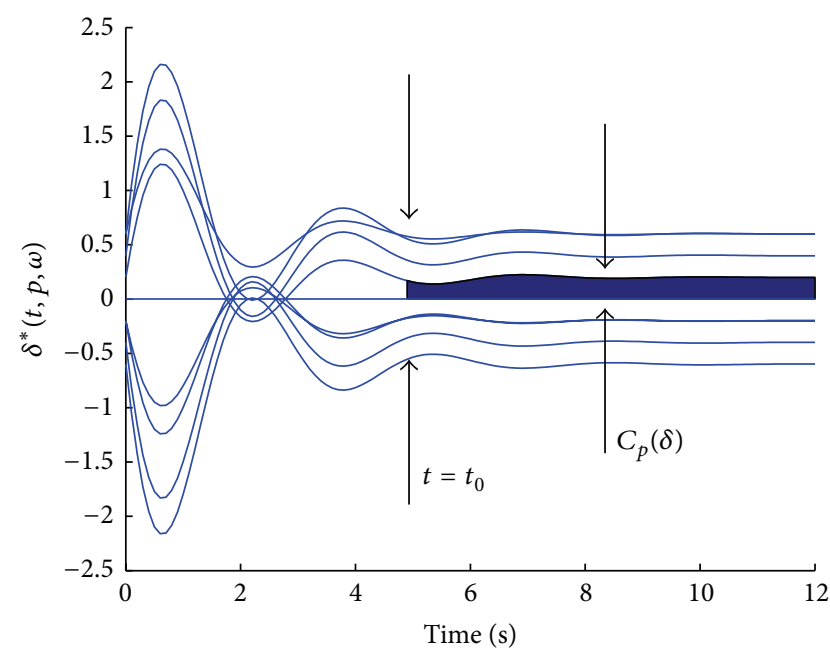

FIGURE 1: Deviation and accumulated angular deviation range.

supports of invariant measures that can occur in nonlinear stochastic systems of the type $\dot{y}=f(y, p)+B \xi_{t}$ do not happen for random perturbations with sufficiently small range, and hence the dynamic behavior of the nonlinear system around $y^{*}$ resembles that of (3) around the origin.

2.2. Indicators of Power System Performance. Theorem 1 shows the existence of a stationary solution for the entire stochastic system (3). In practice, however, the machine variables (rotor angles and velocities) play the major role in system assessment. Note that projections of stationary solution $x^{*}(t, p, \omega)$ on subspaces result in stationary components of the entire system, whose invariant measures are the marginals of $\mu_{p}$ on these subspaces. Let $\left(\delta_{1}, \ldots, \delta_{n}\right)$ be the (relative) rotor angles of the machines in system (1). To simplify notation, in what follows we will also use $\left(\delta_{1}, \ldots, \delta_{n}\right)$ to denote the incremental angles of the linearized stochastic system (3), and $\delta_{i}^{*}(t, p, \omega)$ are the (stationary) trajectories for $i=1, \ldots, n$ under the random excitation $\xi_{t}$.

Our approach to assess the influence of random perturbations on power system performance uses the deviation of the rotor angles from their reference states. More precisely, we will look at the average total deviation of the rotor angles, the range of deviation of these angles, and the loss of generated power per time unit as indicators of system performance.

2.2.1. Deviation of Rotor Angles. The deviation of the rotor angles from the steady state $\delta_{1}=\delta_{2}=\cdots=\delta_{n}=0$ on the time interval $[0, \infty)$ is measured by

$$
d_{i}(p, \omega)=\int_{0}^{\infty}\left|\delta_{i}^{*}(t, p, \omega)\right| d t, \quad i=1, \ldots, n .
$$

Figure 1 shows a typical evolution of a linear system which is stable. In this figure $C_{p}$ is the range of the angle after transient oscillations have faded away. Note that angle deviations are directly related to power losses in the transmission systems which have to be minimized for an efficient operation.
Now the design goal is to minimize the expected value for these deviations, that is,

$$
\min _{p} \mathbb{E}\left(\sum_{i=1}^{n} d_{i}(p)\right)
$$

where the expectation $\mathbb{E}$ is taken with respect to the underlying measure $\mathbb{P}$ of the background noise. In practice, the infinite time interval $[0, \infty)$ has to be replaced by a finite interval $\left[t_{0}, T\right]$, where $t_{0}$ indicates the transient time until the system, from an initial perturbation, reaches the stationary set $C_{p}(\delta)$, the projection of the invariant set $C_{p}$ on the angular coordinates $\delta_{1}, \ldots, \delta_{n}$. According to the ergodic theorem, compare, for example, [7] and we have

$$
\mathbb{P}\left\{\frac{1}{T} \int_{0}^{T} \chi_{A}\left(\delta_{i}^{*}(t, p, \omega)\right) d t \underset{T \rightarrow \infty}{\longrightarrow} \mu_{p}^{i}(A)\right\}=1,
$$

for all $i=1, \ldots, n$, where $A \subset C_{p}^{i}$ is any Borel set in the $\delta_{i}$-component $C_{p}^{i}$, of the invariant set $C_{p}$, and $\chi_{A}(\cdot)$ is the characteristic function of set $A$. So we get

$$
\begin{aligned}
\mathbb{E}\left(\sum_{i=1}^{n} \int_{t_{0}}^{T}\left|\delta_{i}^{*}(t, p, \omega)\right| d t\right) \\
\approx\left(T-t_{0}\right) \sum_{i=1}^{n} \int_{C_{p}^{i} \cap[0, \infty)} y d \mu_{p}^{i}(y) \\
\quad+\left(T-t_{0}\right) \sum_{i=1}^{n} \int_{C_{p}^{i}-\infty, \cap[0)}(-y) d \mu_{p}^{i}(y)
\end{aligned}
$$

as an approximation to (8) on the time interval $\left[t_{0}, T\right]$. In this case (10) corresponds to the shaded area of Figure 1. The right side in (10) is the typical mean value of area under $\left|\delta_{i}^{*}(t, p, \omega)\right|$ trajectories. These elements are obtained by solving the stochastic linear system described in (3), where $\delta_{i}$ vector are chosen.

In practice, expression (10) is evaluated by numerically computing the invariant distribution $\mu_{p}(\delta)$ on $C_{p}$ and then integrating \pm functions with respect to the marginals $\mu_{p}^{i}, i=$ $1, \ldots, n$ of this invariant distribution. Optimal parameter tuning is then achieved by minimizing the results over the appropriate parameter set $p \in \mathbb{R}^{r}$. The invariant distribution $\mu_{p}^{i}$ guarantees existence of $C_{p}$ and allows to compute $\mathbb{E}\left(\sum_{i=1}^{n} \int_{t_{0}}^{T}\left|\delta_{i}^{*}(t, p, \omega)\right| d t\right)$ considering the range of trajectories $\delta_{i}$ in permanent regimen, avoiding computing high numbers of simulations.

2.2.2. Range of Rotor Angles. An approximation to the problem (8) is provided by

$$
\min _{p} \operatorname{diam}\left(C_{p}(\delta)\right)
$$

where $\operatorname{diam}\left(C_{p}(\delta)\right)$ is the diameter of the invariant set in its angular components. Since $C_{p}(\delta)$ is the support of the invariant measure $\mu_{p}(\delta)$, the angular trajectories will 


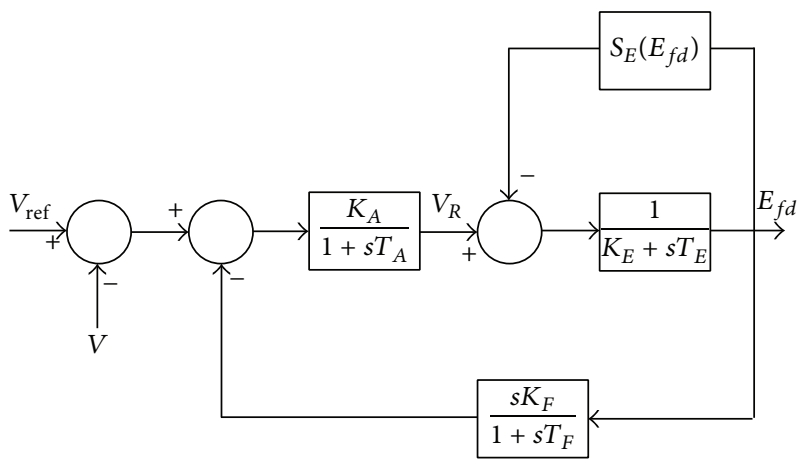

Figure 2: IEEE-Type I exciter model.

reach any open set in $C_{p}(\delta)$ with probability 1 . Therefore $\operatorname{diam}\left(C_{p}(\delta)\right)$ determines the maximum deviation of an angular trajectory from its steady state, while (8) describes the expected deviation. Hereinafter we call $C_{p}$ the "loss range" of the system.

In practice, expression (11) is evaluated by numerically computing the invariant distribution $\mu_{p}(\delta)$ on $C_{p}$ and then integrating the functions \pm with respect to the marginals $\mu_{p}^{i}, i=1, \ldots, n$ of this invariant distribution using (10). Optimal parameter tuning is then achieved by minimizing the results over the appropriate parameter set $p \in \mathbb{R}^{r}$.

2.2.3. Loss of Generated Power. An indicator of system performance that translates directly into financial variables is the loss of generated power due to persistent additive perturbations in a system. Using again the ergodic theorem, the power $\mathrm{Pg}^{i}$ generated per time unit for each machine $i=$ $1, \ldots, n$ can be expressed (in the linearized system (3)) as

$$
P g_{p}^{i}=\int_{C_{p}^{i}} P g(\delta) \mu_{p}^{i}(\delta),
$$

where

$$
P g=I_{d} \cdot V_{i} \cdot \sin \left(\delta_{i}-\theta_{i}\right)+I_{q} \cdot V_{i} \cdot \cos \left(\delta_{i}-\theta_{i}\right),
$$

and

(i) $I_{d}$ and $I_{q}$ are the currents in the $d$ - $q$ axes,

(ii) $V$ is the voltage of the generator bus,

(iii) $\theta$ is the corresponding angle of voltage.

Note. We have chosen Machine 1 as the reference, but it is possible to consider the center of mass.

\section{Power System Model with Additive Random Perturbation}

Let us consider the typical exciter model presented in [4] (see Figure 2).

To illustrate the model let us consider the linearized state equation of a system with constant exciter [4] as follows:

$$
T_{A} \Delta \dot{E}_{f d}=-\Delta \dot{E}_{f d}+K_{A}\left(\Delta V_{\text {ref }}-\Delta V_{t}\right)
$$

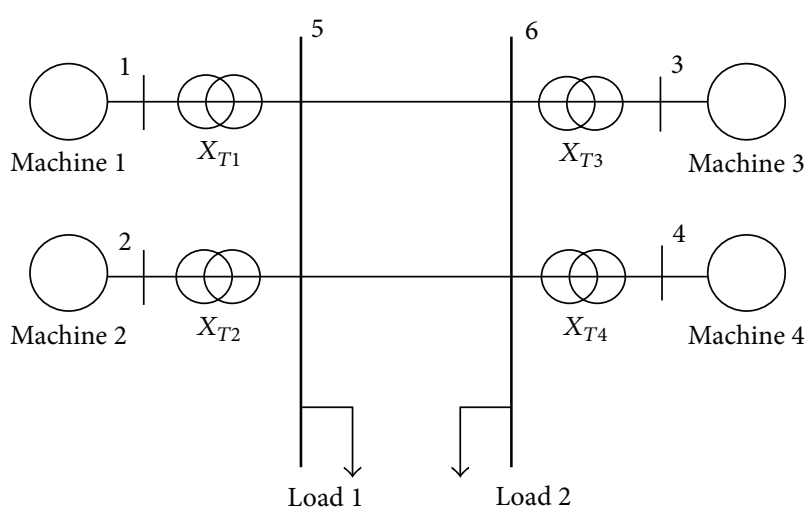

FIGURE 3: Four-machine system.

According to the model presented in [4], we can write the deterministic system as follows:

$$
\dot{x}=A x+E u \text {. }
$$

In classical stability studies with small perturbation, the input vector $\delta u$ is considered null, and therefore the eigenvalues of the matrix of (15) are evaluated.

If it is considered that there are instrumental errors in the telemeasuring systems of the reference voltage of the control system and that those variations are of a random nature and sustained in time, the vector is no longer null. In particular, in this case component $\Delta V_{\text {ref }}$ will not be null and can be written as follows:

$$
\Delta V_{\mathrm{ref}}=\Delta V_{\mathrm{ref}}^{0}+\xi_{t}
$$

where the term $\Delta V_{\text {ref }}^{0}$ corresponds to the situation without perturbation, which in general is assumed to be zero, and $\xi_{t}$ is the stochastic process that represents the measurement errors introduced in the excitation systems of the machines.

According to the above, the linear system (15) is transformed into a stochastic model that takes the form of (3) as follows:

$$
\dot{x}=A x+B u\left(\xi_{t}\right)
$$

The results in Section 5.1 were obtained with the background noise $\eta_{t}$ modeled by a one-dimensional OrnsteinUhlenbeck process (see, e.g., [8] for the properties of this process) as follows:

$$
d \eta_{t}=-\alpha \eta_{t} d t+\beta d W_{t}
$$

where $W_{t}$ is a standard Wiener process and $\alpha$ and $\beta$ are positive constants. (For the results in Section 5.1 the constants are set to $\alpha=\beta=1$.) The random system perturbation is given by

$$
\xi_{t}=\rho \sin \left(\eta_{t}\right) \quad \rho \geq 0
$$

\section{Case Studies}

The results in the following sections were obtained in a fourgenerator two-area system (cf. [5], as shown in Figure 3). 


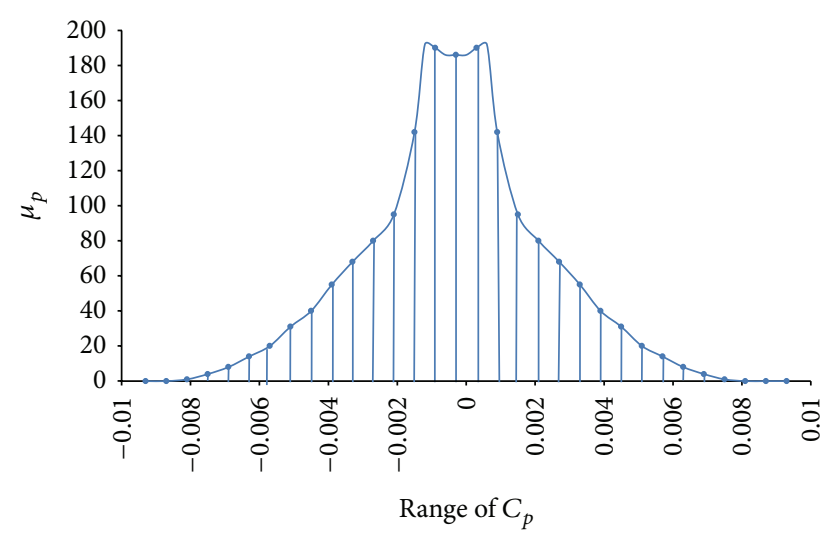

Figure 4: Invariant distribution of generator 2. Perturbation size $\rho=$ 0 .

Machine 1 is the reference where an additive random perturbation is introduced in the frequency signal. Regarding the PSS equipment two cases were considered as follows:

(1) two PSS, one in generator 2 and the other in generator 4,

(2) only one PSS at generator 4.

Parameter $p \in \mathbb{R}^{2}$ in (3) reflects the gain constants of the two PSS, which were set to $k \cdot K_{\text {nom }}$, where $K_{\text {nom }}$ is the nominal value and $k \in\{0.6,0.8,1.0,1.2,1.4\}$.

The perturbation size $\rho \geq 0$ was varied between 0 (for the unperturbed system) and 20.

The purpose of this analysis is to evaluate if, under normal conditions, the parameters of PSS are the optimal.

\section{Results}

The results obtained by applying the proposed methodology to the test systems are given below.

5.1. Invariant Distributions of the Angle Processes $\delta_{i}$. The invariant distributions $\mu_{p}$ of the system (3) are the key objects for the performance assessment of the system under random additive perturbations. The following results show some of the simulated distributions for varying perturbation ranges.

5.1.1. Case 1: PSS at Generators 2 and 4. For Figures 4 and 5 the gain constants of the PSS are set to $1.4 \cdot K_{\text {nom }}$ for generator 2 and to $0.8 \cdot K_{\text {nom }}$ for generator 4 . The figures show the (marginal) invariant distribution of the angular behavior of generator 2 for various perturbation ranges, based on the time interval $[0,30 \mathrm{~s}]$.

The figures show that for increasing perturbation range the distributions become increasingly skewed to the right, and their support increases in size.

5.1.2. Case 2: PSS at Generator 4. For Figures 6 and 7 the gain constant of the PSS is set to $1.4 \cdot K_{\text {nom }}$ for generator 4 , and the simulation time interval is again $[0,30 \mathrm{~s}]$.

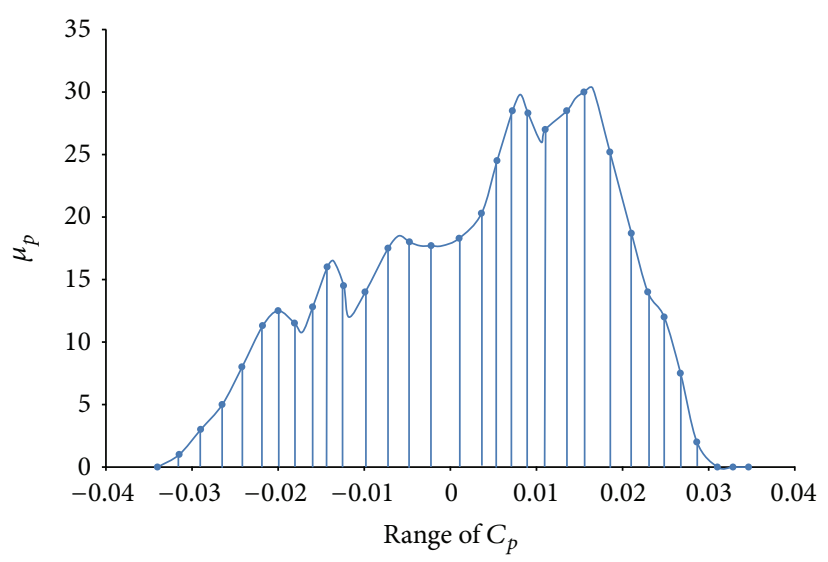

FIGURE 5: Invariant distribution of generator 2. Perturbation size $\rho=$ 5 .

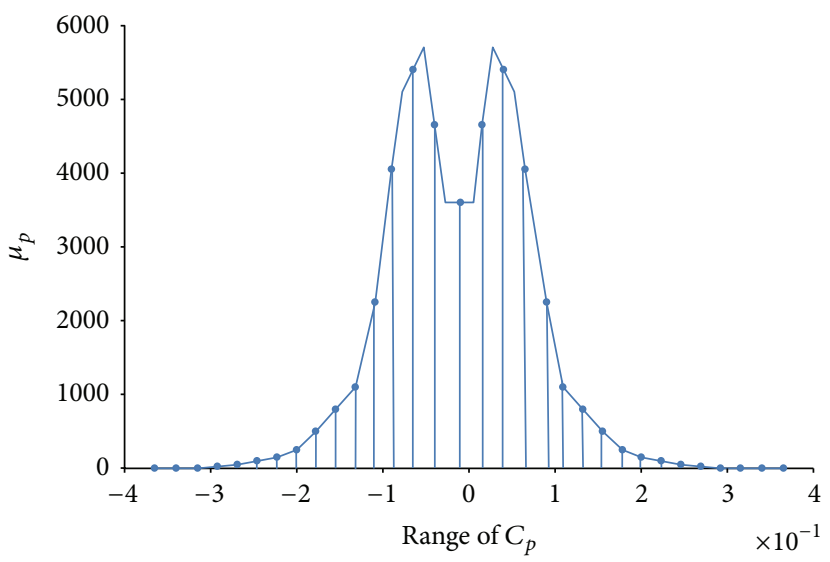

FIGURE 6: Invariant distribution of generator 2. Perturbation size $\rho=$ 0 .

The figures show that for increasing perturbation range the distributions become increasingly skewed to the right, and their support increases in size. Skewness is reduced compared to Case 1 (with PSS at two generators), but the size of the support of the invariant distributions is substantially larger.

5.2. Selection of the Simulation Time Using Case 2. Reliable computation of performance indices for power system behavior using the ergodic theorem depends on the accuracy with which the invariant distribution of the system is computed. Two quantities are crucial when using (10), the simulation interval $[0, T]$ and the choice of the initial value for the computation of the trajectories $\delta_{i}^{*}(t, p, \omega)$. This section shows the dependence of the three indicators on the simulation interval, which was chosen to be $[0,30 \mathrm{~s}]$ in Section 5.2.1 and $[0,120 \mathrm{~s}]$ in Section 5.2.2. The results for 64 initial values were computed and averaged, using 20 simulated trajectories of the background noise $\eta_{t}, t \geq 0$.

The computations use Case 2 (PSS at generator 4 ) with the gain constant factor set to $k \in\{0.6,0.8,1.0,1.2,1.4\}$. 


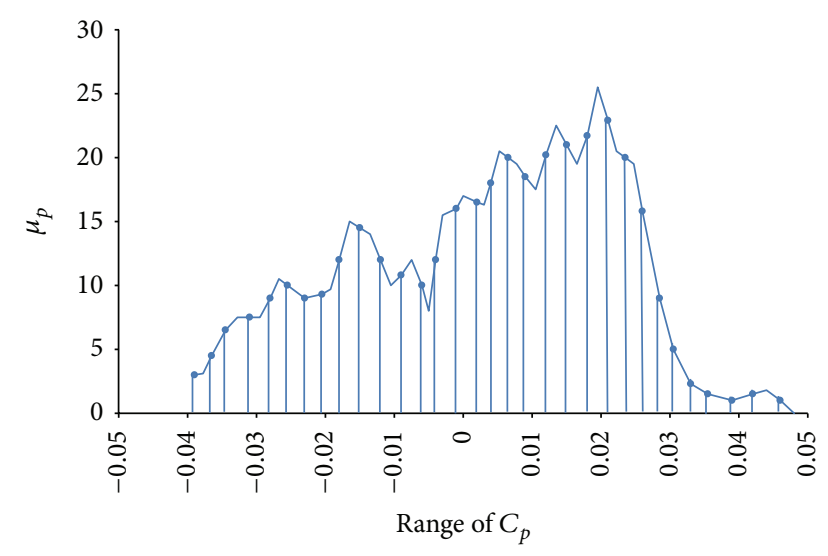

FIGURE 7: Invariant distribution of generator 2. Perturbation size $\rho=$ 5.

TABLE 1: Diameter of the loss range $C_{p}$ for generator 2.

\begin{tabular}{lccccc}
\hline$\rho$ & $k=0.6$ & $k=0.8$ & $k=1.0$ & $k=1.2$ & $k=1.4$ \\
\hline 0 & 0.00001 & 0.0001 & 0.0001 & 0.0001 & 0.0001 \\
5 & 0.0613 & 0.0614 & 0.0615 & 0.0616 & 0.0617 \\
10 & 0.1225 & 0.1227 & 0.1230 & 0.1232 & 0.1233 \\
15 & 0.1995 & 0.1997 & 0.1999 & 0.2001 & 0.2002 \\
20 & 0.2660 & 0.2663 & 0.2665 & 0.2667 & 0.2669 \\
\hline
\end{tabular}

TABLE 2: Total angle deviation for generator 2.

\begin{tabular}{lccccc}
\hline$\rho$ & $k=0.6$ & $k=0.8$ & $k=1.0$ & $k=1.2$ & $k=1.4$ \\
\hline 0 & 0.00003 & 0.00004 & 0.00006 & 0.00007 & 0.0008 \\
5 & 0.01609 & 0.01610 & 0.01610 & 0.01611 & 0.01612 \\
10 & 0.03217 & 0.03219 & 0.03220 & 0.03222 & 0.03223 \\
15 & 0.04656 & 0.04659 & 0.04662 & 0.04665 & 0.04668 \\
20 & 0.06208 & 0.06212 & 0.06216 & 0.06220 & 0.06224 \\
\hline
\end{tabular}

\subsubsection{Simulation Time $30 \mathrm{~s}$}

(i) Size of the loss range $C_{p}^{i}$ (see Table 1 ).

These results show that the loss range increases with the size of the perturbation, and that generator 2 exhibits a larger loss range than the two generators in area 2, where the PSS is located. $C_{p}$ is obtained at $T=30 \mathrm{~s}$.

(ii) Total angle deviation $d_{i}(p)$ (see Table 2).

These results show that the total angle deviation increases with the size of the perturbation and that generator 2 exhibits a larger deviation than the two generators in area 2, where the PSS is located.

(iii) Generated power $P g_{p}^{i}$ (see Table 3).

5.2.2. Simulation Time $120 \mathrm{~s}$. This section shows the simulation results when the time interval is increased to [0,120 s]. $C_{p}$ in this case is obtained at $T=120 \mathrm{~s}$ as follows:

(i) size of the loss range $C_{p}^{i}$ (see Table 4$)$,
TABLE 3: Power generated at machine 2.

\begin{tabular}{lccccc}
\hline$\rho$ & $k=0.6$ & $k=0.8$ & $k=1.0$ & $k=1.2$ & $k=1.4$ \\
\hline 0 & 3.971 & 3.971 & 3.971 & 3.971 & 3.97 \\
5 & 4.053 & 4.052 & 4.052 & 4.052 & 4.052 \\
10 & 4.146 & 4.146 & 4.146 & 4.146 & 4.146 \\
15 & 4.231 & 4.231 & 4.231 & 4.231 & 4.231 \\
20 & 4.319 & 4.319 & 4.319 & 4.319 & 4.319 \\
\hline
\end{tabular}

TABLE 4: Diameter of the loss range $C_{p}$ for generator 2.

\begin{tabular}{lccccc}
\hline$\rho$ & $k=0.6$ & $k=0.8$ & $k=1.0$ & $k=1.2$ & $k=1.4$ \\
\hline 0 & $\approx 0$ & $\approx 0$ & $\approx 0$ & $\approx 0$ & $\approx 0$ \\
5 & 0.0653 & 0.0653 & 0.0654 & 0.0654 & 0.0655 \\
10 & 0.1306 & 0.1307 & 0.1308 & 0.1309 & 0.1310 \\
15 & 0.1959 & 0.1960 & 0.1962 & 0.1964 & 0.1966 \\
20 & 0.2612 & 0.2614 & 0.2616 & 0.2619 & 0.2621 \\
\hline
\end{tabular}

TABLE 5: Total angle deviation for generator 2 .

\begin{tabular}{lccccc}
\hline$\rho$ & $k=0.6$ & $k=0.8$ & $k=1.0$ & $k=1.2$ & $k=1.4$ \\
\hline 0 & $\approx 0$ & $\approx 0$ & $\approx 0$ & $\approx 0$ & $\approx 0$ \\
5 & 0.0147 & 0.0147 & 0.0147 & 0.0147 & 0.0147 \\
10 & 0.0295 & 0.0295 & 0.0295 & 0.0295 & 0.0295 \\
15 & 0.0442 & 0.0442 & 0.0442 & 0.0443 & 0.0443 \\
20 & 0.0589 & 0.0590 & 0.0590 & 0.0590 & 0.0591 \\
\hline
\end{tabular}

TABLE 6: Power generated at machine 2.

\begin{tabular}{lccccc}
\hline$\rho$ & $k=0.6$ & $k=0.8$ & $k=1.0$ & $k=1.2$ & $k=1.4$ \\
\hline 0 & 3.9709 & 3.9705 & 3.9704 & 3.9708 & 3.9709 \\
5 & 4.0552 & 4.0551 & 4.055 & 4.0549 & 4.0547 \\
10 & 4.1414 & 4.1413 & 4.1413 & 4.1412 & 4.1412 \\
15 & 4.2262 & 4.2263 & 4.2263 & 4.2262 & 4.2262 \\
20 & 4.3097 & 4.3098 & 4.3098 & 4.3098 & 4.3098 \\
\hline
\end{tabular}

(ii) total angle deviation $d_{i}(p)$ (see Table 5),

(iii) generated power $\mathrm{Pg}_{p}^{i}$ (see Table 6).

The indices show basically the same behavior depending on factor $k$ of the gain constant and on the perturbation range $\rho$ as for $T=30 \mathrm{~s}$. But note that, for example, the diameter of the loss range is decreased because the transient behavior has died down further at $T=120 \mathrm{~s}$. It appears that for this system a simulation time of $T=30 \mathrm{~s}$ is sufficient to evaluate the different performance indices.

5.3. Analysis of Optimal Gain Tuning Using Case 1. This section summarizes our findings for optimal gain tuning of the PSS in Case 1, that is, two PSS, one in generator 2 and the other in generator 4 . The optimization criterion uses formula (12), measuring the power generated at each of the machines. The results are based on a simulation time of $T=30 \mathrm{~s}$.

In Table $7 k_{2}$ and $k_{4}$ are the factors of the gain constants of the PSS at generators 2 and 4 , respectively. 
TABLE 7: Average power generated at machines 2-4.

\begin{tabular}{lllllll}
\hline$k_{2}$ & $k_{4}$ & $\rho=0$ & $\rho=5$ & $\rho=10$ & $\rho=15$ & $\rho=20$ \\
\hline 0.6 & 0.6 & 4.2571 & 4.3195 & 4.3850 & 4.4525 & 4.5195 \\
0.8 & 0.6 & 4.2574 & 4.3213 & 4.3886 & 4.4552 & 4.5216 \\
1.0 & 0.6 & 4.2578 & 4.3204 & 4.3866 & 4.4528 & 4.5184 \\
1.2 & 0.6 & 4.2581 & 4.3189 & 4.3843 & 4.4497 & 4.5143 \\
1.4 & 0.6 & 4.2584 & 4.3176 & 4.3821 & 4.4465 & 4.5102 \\
0.6 & 0.8 & 4.2573 & 4.3217 & 4.3872 & 4.4539 & 4.5205 \\
0.8 & 0.8 & 4.2577 & 4.3230 & 4.3900 & 4.4569 & 4.5233 \\
1.0 & 0.8 & 4.2580 & 4.3213 & 4.3871 & 4.4533 & 4.5188 \\
1.2 & 0.8 & 4.2584 & 4.3197 & 4.3846 & 4.4497 & 4.5142 \\
1.4 & 0.8 & 4.2586 & 4.3183 & 4.3824 & 4.4465 & 4.5100 \\
0.6 & 1.0 & 4.2577 & 4.3220 & 4.3875 & 4.4548 & 4.5217 \\
0.8 & 1.0 & 4.2580 & 4.3233 & 4.3908 & 4.4579 & 4.5242 \\
1.0 & 1.0 & 4.2583 & 4.3219 & 4.3885 & 4.4547 & 4.5201 \\
1.2 & 1.0 & 4.2587 & 4.3204 & 4.3860 & 4.4512 & 4.5156 \\
1.4 & 1.0 & 4.2587 & 4.3191 & 4.3838 & 4.4480 & 4.5114 \\
0.6 & 1.2 & 4.2579 & 4.3215 & 4.3869 & 4.4539 & 4.5207 \\
0.8 & 1.2 & 4.2583 & 4.3228 & 4.3902 & 4.4571 & 4.5234 \\
1.0 & 1.2 & 4.2586 & 4.3214 & 4.3879 & 4.4539 & 4.5192 \\
1.2 & 1.2 & 4.2590 & 4.3201 & 4.3856 & 4.4505 & 4.5147 \\
1.4 & 1.2 & 4.2589 & 4.3190 & 4.3834 & 4.4473 & 4.5105 \\
0.6 & 1.4 & 4.2571 & 4.3210 & 4.3862 & 4.4532 & 4.5198 \\
0.8 & 1.4 & 4.2579 & 4.3222 & 4.3896 & 4.4564 & 4.5224 \\
1.0 & 1.4 & 4.2585 & 4.3210 & 4.3874 & 4.4532 & 4.5183 \\
1.2 & 1.4 & 4.2591 & 4.3198 & 4.3850 & 4.4497 & 4.5137 \\
1.4 & 1.4 & 4.2576 & 4.3186 & 4.3829 & 4.4466 & 4.5096 \\
\hline & & & & & &
\end{tabular}

In order to obtain the optimum tuning for the gain constants $k_{2}$ and $k_{4}$, which should take into account the overall system performance, we average the results over the three machines, as shown in Table 7.

This table shows that optimal system tuning depends strongly on the size of the random perturbation.

(1) For the unperturbed system $(\rho=0)$, optimal performance is accomplished for $k_{2}=0.6$ and $k_{4}=$ 0.6 or 1.4 .

(2) For all perturbation levels $\rho>0$, the optimal setting for the gain constant of the PSS at machine 2 is $k_{2}=$ 1.4. For relatively small noise levels ( $\rho=5$ or 10 ), the optimal setting for the gain constant of the PSS at machine 4 is $k_{4}=0.6$.

(3) As the perturbation level increases, optimality is achieved with larger gain constants for $k_{4}$ : at level $\rho=15, k_{4}=0.6$ and 0.8 perform equally well and the performance for $k_{4}=1.4$ is very close. As the random perturbation increases further to $\rho=20$, optimality in the range studied here is achieved for $k_{2}=1.4$ and $k_{4}=1.4$.

\section{Conclusions}

Several performance indices for power systems with additive random perturbations are presented. These indices are based on the (unique) invariant distribution of the system under Markovian noise. They measure the loss range, total angular deviation, and generated power for each machine of a power system. To obtain an optimality criterion that can be used to tune system parameters, the use of system performance is proposed and averaged over all machines.

The four-generator two-area test system is analyzed in some detail under normal operating conditions, with PSS at generators 2 and 4, or only at generator 4. Using a random perturbation that affects the settings of generator 1 , the power generation criterion is used to obtain optimal settings for the gain constants of the PSSs at generators 2 and 4 . It turns out that optimal tuning of the gain constants depends strongly on the size of the random perturbation; in particular, the optimal settings for noisy systems is different from that for the unperturbed system.

To show the conceptual ideas leading to the new performance indices, the test system is used under normal operating conditions. We expect that the same system under stressed conditions will show even larger differences in performance depending on parameter settings and noise characteristics.

This paper considers power systems under additive random perturbation, as they occur, for example, when external reference signals display random variation. Hence the methodology developed here will allow us to introduce stochastic analysis into the framework of supervisory control when random behavior is present.

\section{Appendix}

Proof of Theorem 1. Under condition 2 the associated linear control system $\dot{x}=A(p) x+B u$ has a unique control set $C_{p}$ with nonvoid interior and $0 \in \operatorname{int} C_{p}$ (see $\left.[6,9]\right)$. Under condition 1 set $C_{p}$ is compact and invariant [6, page 61]. By [10-12] this implies, under conditions 3 and 4, that stochastic system (3) has a unique stationary and ergodic solution $x^{*}(t, p, \omega)$, whose invariant distribution $\mu_{p}$ has as support the set $C_{p}$. This proves part (1). Part (2) follows directly from [12] using the exponential stability of $A(p)$.

\section{Conflict of Interests}

The authors declare that there is no conflict of interests regarding the publication of this paper.

\section{Acknowledgment}

This research was financed by Project Fondecyt 11130169 (Comision Nacional de Investigacion Cientifica y Tecnologica Chile).

\section{References}

[1] D. Q. Wei and X. S. Luo, "Noise-induced chaos in singlemachine infinite-bus power systems," EPL, vol. 86, no. 5, Article ID 50008, 2009.

[2] K. A. Loparo and G. L. Blankenship, "A probabilistic mechanism for small disturbance instabilities in electric power systems," 
IEEE Transactions on Circuits and Systems, vol. 32, no. 2, pp. 177184, 1985.

[3] C. L. De Marco and A. R. Bergen, "A security measure for random load disturbances in nonlinear power system models," IEEE Transactions on Circuits and Systems, vol. 34, no. 12, pp. 1546-1557, 1987.

[4] M. A. Pai, D. P. S. Gupta, and K. R. Padiyar, Small Signal Analysis of Power Systems, reprinted edition, Alpha Science, 2005.

[5] P. Kundur, Power System Stability and Control, McGraw-Hill, New York, NY, USA, 1994.

[6] F. Colonius and W. Kliemann, The Dynamics of Control, Systems \& Control: Foundations \& Applications, Birkhäuser, Boston, Mass, USA, 2000.

[7] K. B. Athreya and S. N. Lahiri, Measure Theory and Probability Theory, Springer Texts in Statistics, Springer, New York, NY, USA, 2006.

[8] W.-C. Xie, Dynamic Stability of Structures, Cambridge University Press, 2006.

[9] E. D. Sontag, Mathematical Control Theory, Springer, New York, NY, USA, 2nd edition, 1998.

[10] W. Kliemann, "Recurrence and invariant measures for degenerate diffusions," The Annals of Probability, vol. 15, no. 2, pp. 690707, 1987.

[11] L. Arnold and W. Kliemann, "On unique ergodicity for degenerate diffusions," Stochastics, vol. 21, no. 1, pp. 41-61, 1987.

[12] F. Colonius and W. Kliemann, "Topological, smooth, and control techniques for stochastic systems," in Stochastic Systems, H. Crauel and M. V. Gundlach, Eds., pp. 181-208, Springer, Berlin, Germany, 1999. 


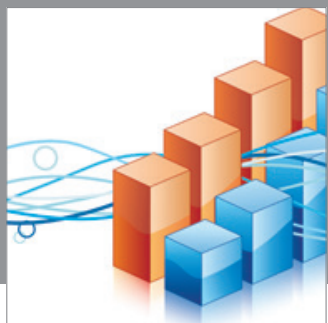

Advances in

Operations Research

mansans

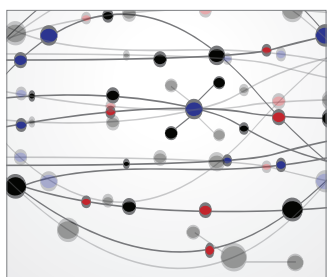

The Scientific World Journal
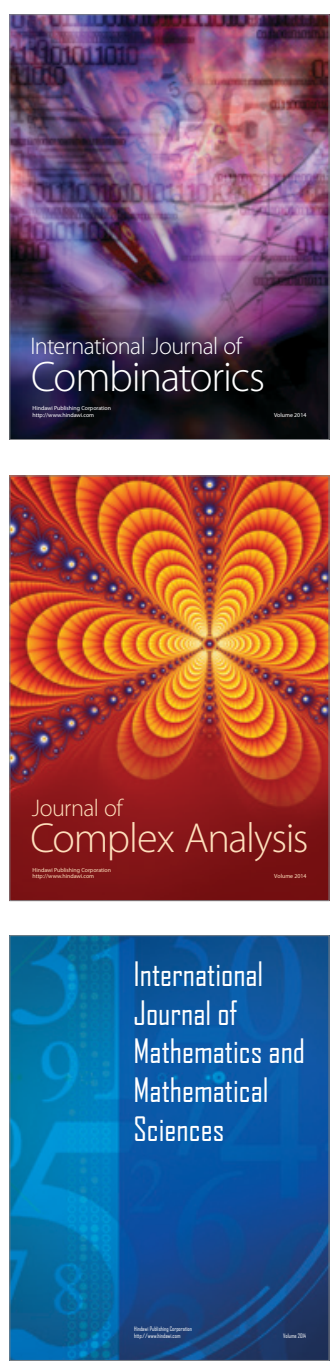
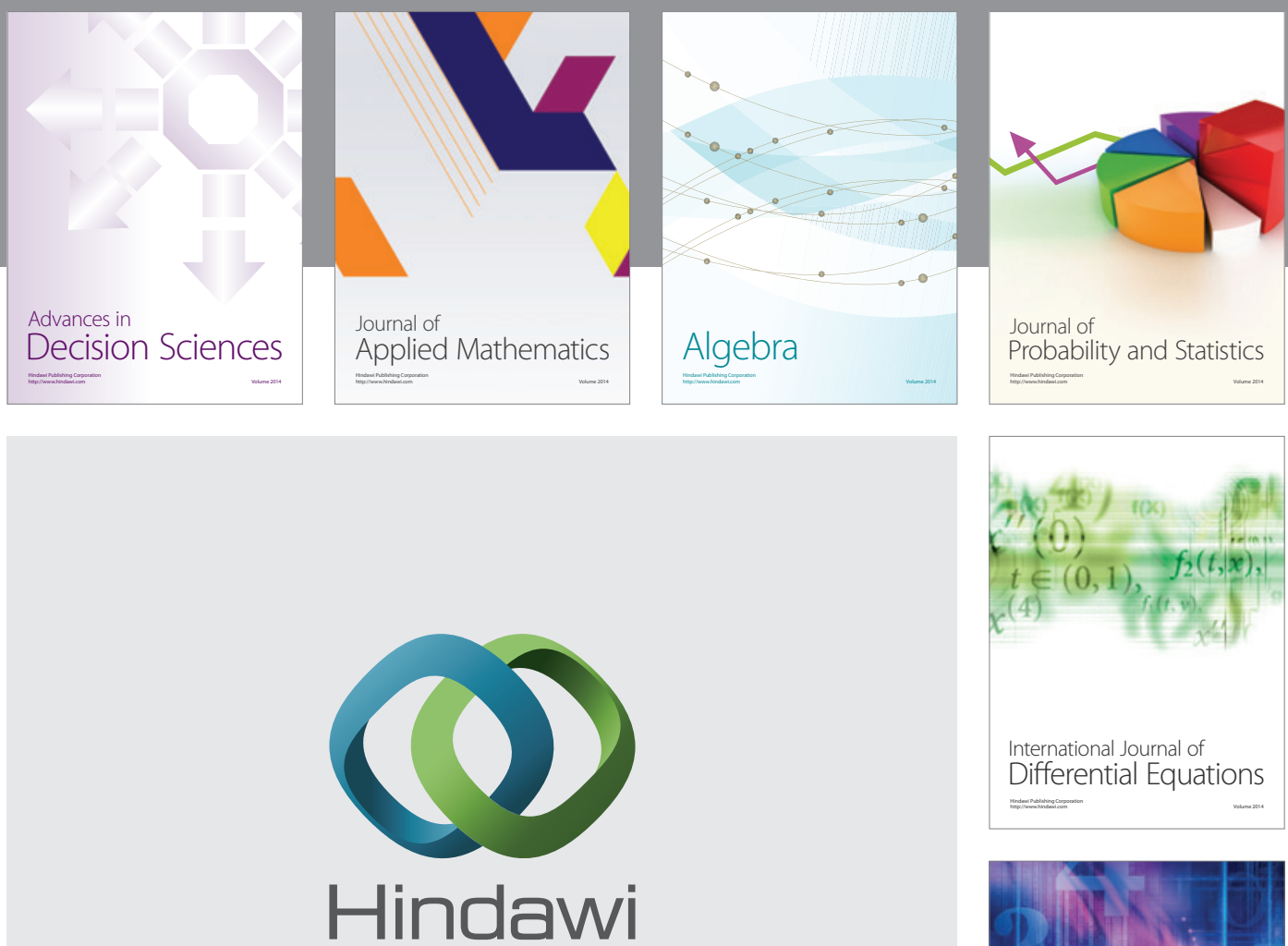

Submit your manuscripts at http://www.hindawi.com
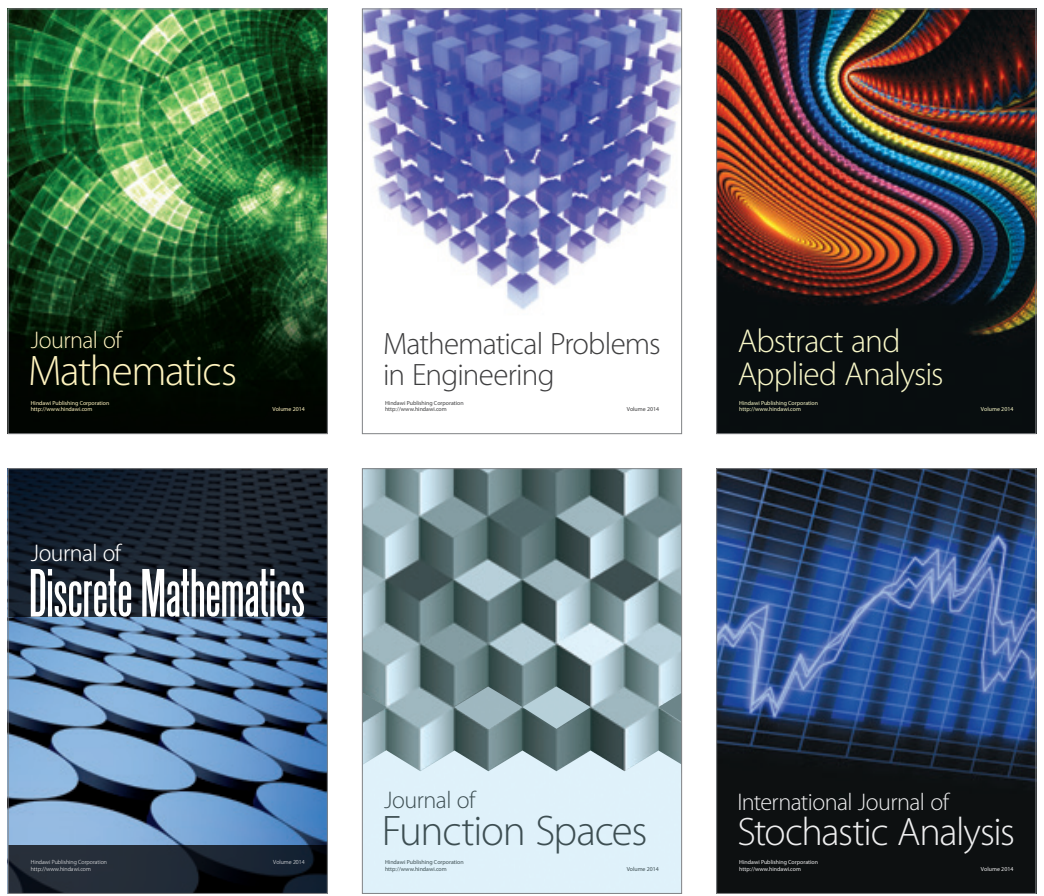

Journal of

Function Spaces

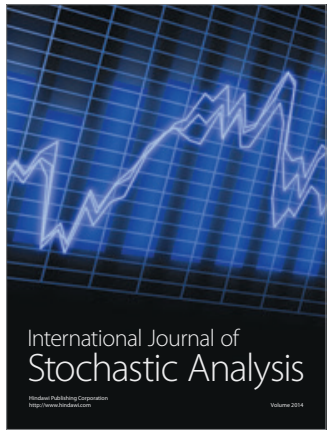

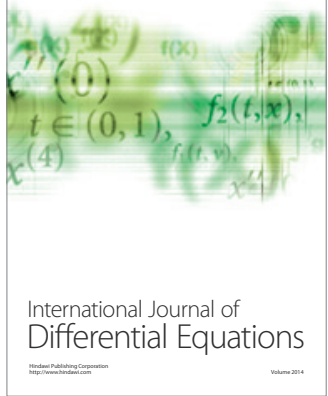
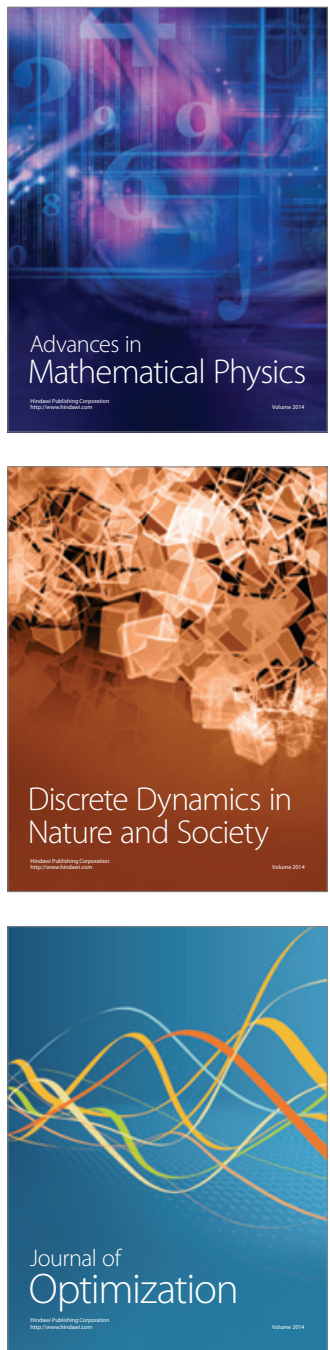